Psychology of Language and Communication 2017, Vol. 21, No. 1

DE GRUYTER

OPEN

DOI: $10.1515 /$ plc-2017-0004

EMILY E. RUSSELL

Department of Child and Adolescent Development, California State University, Northridge

\title{
CHILDREN'S LABEL-LEARNING EXPERIENCE WITHIN SUPERORDINATE CATEGORIES FACILITATES THEIR GENERALIZATION OF LABELS FOR ADDITIONAL CATEGORY MEMBERS
}

\begin{abstract}
In two studies, we tested the relationship between children's label-learning experience and label-learning ability within diverse superordinate categories with complex perceptual organization (animals, clothing, foods). Using both quasi-experimental and experimental designs, we examined 18- and 24-month-old children's ability to generalize labels for novel members of superordinate categories as a product of their previous experience in learning labels for members of those categories. As predicted, children properly generalized more labels for members of the categories within which they had more label-learning experience than for members of the categories within which they had less label-learning experience. Results are consistent with the idea that children develop category-specific label-learning biases through their experience in learning labels for category members; they carry implications for multiple accounts of vocabulary acquisition and identify directions for future research.
\end{abstract}

Key words: label learning, vocabulary acquisition, categorization

\section{Introduction}

To learn the label of an object, a child must determine how the label maps on to the object and how it should be generalized to other objects. For example, if a child hears "spoon" applied to a silver metal spoon, they must decide if the label refers to the object's shape, material, color, or another perceptual or functional feature-essentially, they must decide what makes a spoon a "spoon". Eventually, they must also decide if the label should be applied to a brown wooden spoon

Address for correspondence: Dr. Emily E. Russell, Department of Child and Adolescent Development, California State University Northridge, 280E Sequoia Hall, 18111 Nordhoff Street, Northridge, CA 91330. E-mail: emily.russell@csun.edu 
or a blue plastic spoon. Determining the features of an object that are relevant to its labeling can be a cumbersome task: Labels can refer to objects in many different ways (Quine, 1960). Nevertheless, older children (and adults) excel at it. They can properly map labels to objects after just a few labeling instances (Clark, 1993; Rice, 1980; Soja et al., 1991); for this reason, they learn new labels quickly. In contrast, younger children often require many labeling instances before they properly map a label to an object (Rice, 1980); even after they have seemingly learned the label, they might over-generalize it or misapply it (Elbers, 1995; Gershkoff-Stowe \& Smith, 1997). Not until 18 months of age do most children demonstrate accurate and efficient mapping behavior (Bloom, 1973; Carey \& Bartlett, 1978; Clark, 1993; Nelson, 1973; Gershkoff-Stowe \& Smith, 1997; Soja, Carey, \& Spelke, 1991). The pace of their vocabulary acquisition, initially leisurely, begins to accelerate (Bloom, 1973; Carey \& Bartlett, 1978; Clark, 1993; Nelson, 1973; Gershkoff-Stowe \& Smith, 1997; Soja, Carey, \& Spelke, 1991).

Why does children's label-learning ability improve over time? A large body of research (e.g. Gershkoff-Stowe \& Hahn, 2007; Gershkoff-Stowe \& Smith, 2004; Mervis \& Bertrand, 1994/1995; Smith, Jones, Landau, Gershkoff-Stowe, \& Samuelson, 2002; Thom \& Sandhofer, 2009) suggests that children's labellearning experience-that is, the number and type of labels that children have previously learned-predicts their mapping behavior, and, in turn, their labellearning ability. This relationship is particularly strong when the labels that children have previously learned, and the labels that they are trying to learn, refer to categories of objects with similar perceptual organization (Gershkoff-Stowe \& Smith, 2004; Smith et al., 2002; Thom \& Sandhofer, 2009). For example, the more labels for objects from shape-based categories (i.e. categories in which shape is common among members, e.g. the category of objects labeled by "ball") that children know, the more frequently they map novel objects' labels by shape, and the more labels for objects from shape-based categories they subsequently learn (Gershkoff-Stowe \& Smith, 2004; Smith et al., 2002). Similar findings have been documented for color-based object categories (Thom \& Sandhofer, 2009). Thus, children's label-learning ability depends, in part, on the extent and nature of their label-learning experience. Through their label-learning experience, they appear to develop strategies for determining the features of objects that are relevant to their labeling.

The majority of previous work has focused on the relationship between children's label-learning experience and label-learning ability for object categories with similar perceptual organization, regardless of the superordinate category to which they belong; thus, their findings can only speak to how children develop category-general label-learning strategies. Although children's mapping behavior is initially quite undifferentiated, it begins to vary between superordinate categories over time (Booth \& Waxman, 2002; Clark, 1973; Graham \& PoulinDubois, 1999; Imai, Gentner, \& Uchida, 1994; Jones \& Smith, 1998/2002; Jones et al., 1991; Keil, 1994; Lavin \& Hall, 2002; Macario, 1991; Samuelson et al., 
2008; Santos et al., 2002). This suggests that they develop multiple label-learning strategies, specific to each superordinate category (Jones \& Smith, 1998/2002; Lavin \& Hall, 2002). In the current studies, we sought to better understand how these category-specific label-learning strategies develop. In other words, we explored the factors that facilitate children's label learning within, rather than across, superordinate categories. We examined children's label-learning ability as a product of their label-learning experience within superordinate categories with diverse perceptual organization.

\section{Label-learning experience promotes the development of label-learning strategies}

Despite their initial struggles, most children begin exhibiting more accurate and efficient mapping behavior at around 18 months of age (Clark, 1993; Rice, 1980; Soja et al., 1991). Around this same time, many children experience a period of rapid vocabulary growth, during which their noun vocabulary sizes might double or triple in the span of just a few months (Bates et al., 1988; Bloom, 1973; Clark, 1993; Nelson, 1973). The onset of this period has been linked to the development of the shape bias: the well-documented tendency of children to map object labels by shape rather than by other perceptual features that are equally plausible as referents (Clark, 1973; Graham \& Poulin-Dubois, 1999; Hupp, 2015; Imai et al., 1994; Jones et al., 1991; Landau, Smith, \& Jones, 1988; Samuelson \& Horst, 2007 but cf. Woodward, Markman, \& Fitzsimmons, 1994).

There are a number of theoretical conceptualizations of the shape bias, but most agree that it facilitates label learning by serving as a heuristic for determining how labels refer to objects (Booth, Waxman, \& Wang, 2005; Diesendruck, Markson, \& Bloom, 2003; Markman, 1991; Smith, 2000/2001). Parents frequently label objects from shape-based categories when speaking to their young children (Sandhofer, Smith, \& Luo, 2000); thus, from a child's perspective, shape has been relevant to labeling and will likely continue to be in the future. Mapping object labels by shape is not a perfect label-learning strategy, but it is, perhaps, the optimal one.

The shape bias increases in strength as children amass label-learning experience (Gershkoff-Stowe \& Smith, 2004; Samuelson \& Smith, 1999; Smith et al., 2002). Over multiple sessions, Smith et al. (2002) trained 17-month-old children in labels for numerous novel objects from experimentercreated categories that were based purely on shape (i.e. shape was the only feature common among members). At the end of training, they measured the frequency with which children generalized labels of untrained novel objects by shape, color, or material. Results indicated that children generalized labels by shape more frequently than by material or color-and that this tendency was more pronounced in children in the experimental group than in children in an untrained control group. Additionally, in the months following training, children in the experimental group experienced accelerated vocabulary acquisition within 
shape-based object categories outside of the laboratory (children in the control group did not). The authors' findings suggest that: a) children's experience in learning labels for objects from shape-based categories increased the strength of their shape bias, and $b$ ) the shape bias promotes acquisition of labels for objects from shape-based categories. In other words, they suggest that the shape bias is one factor driving the relationship between children's label-learning experience and label-learning ability.

Importantly, the relationship between children's label-learning experience and their label-learning ability also exists for object categories organized by non-shape perceptual features (Gershkoff-Stowe \& Hahn, 2007; Jones \& Smith, 2002; Jones et al., 1991; Smith, 2000/2001; Thom \& Sandhofer, 2009). Thom and Sandhofer (2009) trained 20-month-old children with very limited color vocabularies in two, four, or six color labels. Over the course of ten training sessions, they exposed children to multiple objects in each color in their training set and applied the color labels to those objects numerous times. They then tested children's ability to generalize new, previously untrained color labels. They found that children who were trained in six color labels properly generalized more color labels than children who were trained in two or four color labels; additionally, only children who were trained in six color labels performed at levels above what would be expected by chance. The researchers argued that children in the six-color label condition had developed a "color bias", which facilitated their acquisition of additional color labels.

Taken together, the findings of Smith et al. (2002) and Thom and Sandhofer (2009) suggest that children develop label-learning strategies for shape-based object categories and color-based object categories through their label-learning experience. Furthermore, the findings of Thom and Sandhofer emphasize that the amount of label-learning experience matters: Children's ability to learn labels for category members increases in proportion to the number of labels for category members they have previously learned. Other studies have also provided evidence of this phenomenon for both color-based and shape-based categories (e.g. Gershkoff-Stowe \& Hahn, 2007; Gershkoff-Stowe \& Smith, 2004; Samuelson, 2000, Sandhofer \& Smith, 1999).

Why label-learning experience matters. We, like others (e.g. Smith \& Samuelson, 2006; Smith et al., 2001; Smith, 2000/2001), believe that children develop label-learning strategies via their experience because it exposes them to the perceptual features correlated among members of object categories. For example, children develop the shape bias because members of object categories frequently have similar shapes (Samuelson \& Smith, 1999). Sensitive to these regularities, children learn to associate the shape of objects with the act of labeling-an association that is strengthened with every new label for an object from a shape-based category that they learn (Jones et al., 1991; Jones \& Smith, 2002; Smith, 2000/2001; Smith et al., 2002). Eventually, they begin to attend automatically to object shape in label-learning contexts, making them 
more likely to map labels by that feature. Children develop a color bias in a similar manner-as long as their label-learning experience has provided them with sufficient exposure to color correlations among members of object categories (e.g. as in Thom \& Sandhofer, 2009).

\section{The diversity of object categories}

The shape bias and color bias might promote children's label learning in many instances, but not in all (Bloom, 1973; Bowerman, 1978). Although categories of solid, man-made objects (i.e. artifacts) tend to be organized by shape, categories of deformable objects tend to be organized by material (Carey, 1978; Gelman, 1988; Imai \& Gentner, 1997; Samuelson, Horst, Schutte, \& Dobbertin, 2008; Samuelson \& Smith, 1999; Smith, 2000; Soja et al., 1991; Subrahmanyam et al., 1999). There is even greater variability in perceptual organization between superordinate categories-that is, the broad, higher-level categories (e.g. animals, foods, clothing, etc.) that encompass specific, basic-level categories of objects (e.g. cats, apples, shirts, etc.). For example, previous research (e.g. Booth \& Waxman, 2002; Jones \& Smith, 2002; Jones et al., 1991; Shutts, Markson, \& Spelke, 2009; Waxman \& Huang, 2005) suggests that the superordinate category of animals is organized by shape and texture, meaning it encompasses numerous basic-level categories of shape-similar objects and/or texture-similar objects. Other perceptual features might be relevant to labeling within the basic or subordinate categories nested within the superordinate category of animals. However, shape and texture appear to be the most common perceptual features across all instances of animals. For example, color might be relevant to labeling within the basic-level category of zebras, but it is less relevant to labeling horses, dogs, birds, and so forth. In a similar way, the superordinate category of foods is organized by color and material, meaning it encompasses numerous basic-level categories of color-similar objects and/or material-similar objects (Lavin \& Hall, 2002; Macario, 1991; Santos, Hauser, \& Spelke, 2002). Thus, children cannot effectively rely on any single strategy-a shape bias, color bias, or otherwise-to learn labels for objects from all basic or superordinate categories.

Although young children often over-map and over-generalize object labels by shape (Clark, 1973; Graham \& Poulin-Dubois, 1999; Jones et al., 1991; Jones \& Smith, 1998; Samuelson et al., 2008), their mapping behavior becomes more nuanced over time (Booth \& Waxman, 2002; Imai, Gentner, \& Uchida, 1994; Jones \& Smith, 2002; Jones \& Smith, 1998; Jones et al., 1991; Keil, 1994; Lavin \& Hall, 2002; Macario, 1991; Samuelson et al., 2008; Santos et al., 2002). For example, Lavin and Hall (2001) demonstrated differences in threeyear-old children's mapping and generalization of labels for toys and foods. The researchers manipulated children's understanding of labeled novel objects' superordinate categories by saying that they liked to either "play with" them or "eat" them. In some instances, the objects were solid (e.g. made of wax); in other instances, the objects were non-solid (e.g. made of Vaseline). They 
then asked children to choose another referent of the label from a set of two test objects: one that matched the target object in material, but not in shape, and one that matched the target object in shape, but not color, texture, or smell. They found that children generalized the labels of toys more frequently by shape than they did the labels of foods. And, although children generalized the labels of solid objects by shape more frequently than they did the labels of non-solid objects, this pattern was less pronounced for the labels of foods.

Thus, by about three years of age, children appear to utilize multiple label-learning strategies, each seemingly specific to a superordinate category. What are these strategies and how do they develop? It has been proposed that children's label-learning strategies become more numerous and nuanced over time because their linguistic experience increases, and with it, their exposure to the correlated perceptual features among members of superordinate categories (Jones \& Smith, 2002; Jones et al., 1991; Smith, 2000/2001; Thom \& Sandhofer, 2009). Children's attention eventually tunes to these perceptual features in label-learning contexts just as it does to shape (Jones \& Smith, 2002; Jones et al., 1991; Smith, 2000/2001). And, just like the shape bias, these categoryspecific attentional biases facilitate label learning by helping children determine how labels refer to new category members. It is proposed that this process can occurs for any superordinate category, regardless of its perceptual organization (Smith, 2000). We echo these predictions, and also propose that these attentional biases develop and function independently within each superordinate category. Given the diversity of perceptual organization between superordinate categories, an attentional bias within one superordinate category should only result from children's previous experience in learning labels for its members; additionally, it should only facilitate their subsequent learning of labels for its members.

Importantly, preliminary support for these predictions comes from Jones and Smith (2002). First, they analyzed the content of children's typical vocabularies within the superordinate category of animals and the class of artifacts, which encompasses the superordinate categories of furniture, small household items, vehicles and the like. They found that between the superordinate category of animals and the class of artifacts, children knew a comparable number of labels for shape-based object categories; however, they knew more labels for texture-based categories in the superordinate category of animals and in the class of artifacts. From this, the researchers argued that the words children learn within the superordinate category of animals contain regularities that might promote a bias to map and generalize novel objects' labels by shape and texture. Next, they trained children in labels for a few animal-like novel objects. These objects had cues to animacy (i.e. eyes), and their shape and texture were emphasized during training. The researchers then tested children's generalization of labels for additional animal-like novel objects and artifact-like novel objects with no cues to animacy. They labeled novel objects for children and asked them if the labels also referred to test objects that matched the target object in shape, texture, 
or shape and texture. They found that children generalized the labels of the "animals" by shape and texture but the labels of the "artifacts" by shape alone.

\section{Current studies and hypotheses}

The findings of Jones and Smith (2002) suggest that children are capable of forming attentional biases within a superordinate category via their labellearning experience-and that an attentional bias within one superordinate category (i.e. animals) does not facilitate label learning within other classes or superordinate categories (i.e. artifacts). However, it is yet unknown if the relationship between children's label-learning experience and label-learning ability also exists within other superordinate categories-such as those that are not animate, or not organized, at least in part, by shape. It is possible that the shape bias provides an essential foundation which is additional to label-learning biases. For example, children might only develop a shape-and-texture bias specific to the superordinate category of animals because they originally possessed a more generalized shape bias. Conversely, the shape bias might inhibit children from developing label-learning biases within superordinate categories that are not based on shape (Samuelson et al., 2008; Sandhofer \& Smith, 2007). Additionally, it is yet unknown if this relationship exists outside of the laboratory-for superordinate categories that are not carefully controlled by experimenters. It is possible that real superordinate categories do not contain the perceptual regularities necessary for children to form label-learning strategies. For example, within the superordinate category of "animals" created by Jones and Smith (2002), both shape and texture perfectly predicted membership. That is, shape and texture were common among all members. However, their analysis of the content of children's vocabularies revealed only that they typically know numerous labels for shape-based object categories and texture-based object categories; it did not specify how many of these labels refer to object categories based on both shape and texture. More detailed analyses of the content of children's typical vocabularies (e.g. Russell \& Kyger, under review) suggest that no perceptual feature, or set of perceptual features, perfectly predicts membership to the superordinate category of animals (or to any superordinate category).

In two studies, we addressed these remaining questions in order to provide additional support to our general predictions. In Study 1, we examined children's ability to generalize labels for rare members of the superordinate categories of animals, clothing items, and foods as a product of their vocabulary sizes within each superordinate category, which we considered as proxy measures of their label-learning experience. In Study 2, we experimentally manipulated the amount of children's label-learning experience within the superordinate categories of animals and clothing items and tested their ability to generalize labels for additional rare members of each superordinate category. Based on the findings of previous work (e.g. Gershkoff-Stowe \& Smith, 2004; Jones \& Smith, 2002; Thom \& Sandhofer, 2009), we hypothesized that, in both studies, children 
would correctly generalize more labels for members of the superordinate categories within which they had more label-learning experience than for members of the superordinate categories within which they had less labellearning experience. We included the superordinate category of animals to be consistent with similar studies (e.g. Jones et al., 1991; Jones \& Smith, 2002). We included the superordinate category of clothing items because it belongs to the class of artifacts, and appears to be based on shape and material (Russell $\&$ Kyger, under review). Finally, we included the superordinate category of foods because it is based on material and/or color (Lavin \& Hall, 2001; Macario, 1991). These latter choices of superordinate categories allowed us to determine how widely relevant phenomenon extend. Furthermore, using children's existing vocabulary size to measure their label-learning experience and real members of the superordinate categories during training and testing ensured the ecological validity of our findings.

Alternative frameworks. It should be acknowledged that if our hypotheses are supported, our findings would also be consistent with a competing conceptualization of children's label-learning strategies, one positing that children's mapping behavior is influenced by their ontological understanding of the categories (e.g. Bloom, 2000; Booth \& Waxman, 2002a/b; Booth et al., 2005; Diesendruck \& Bloom, 2003; Diesendruck et al., 2003; Gelman, 1988; Keil, 1994; Mandler \& McDonough, 1998). Its proponents argue that children exhibit a shape bias "not because of a direct correlation with categories, but because [shape] is causally related to deeper conceptual qualities of objects from a broad range of ontological categories" (Booth \& Waxman, 2002a; p. B13; see also Bloom \& Markson, 1998; Soja et al., 1991). The shape bias increases in strength as children gain understanding of object categories (Booth \& Waxman, 2002b). Their label-learning strategies increase in number and diversify because their understanding of each superordinate category increases (Diesendruck \& Bloom, 2003; Diesendruck et al., 2003; Kemler Nelson, 1995). For example, they learn what it means for something to be a toy versus a food. Although we favor one interpretation over another, our objective was to establish that the relationship between children's label-learning experience and label-learning ability exists within superordinate categories-not to determine which of these interpretations, or others, is most accurate. The value of the current studies lies in the fact that our findings carry implications for multiple theoretical frameworks of children's vocabulary acquisition.

\section{Study 1}

\section{Method}

Participants. Fourteen children (seven males) participated in Study 1. On average, they were 18.35 months old $(S D=1.15$, range $=17.50-21.20)$ and knew 131.67 words $(S D=167.85$, range $=9-500)$ in total. All children were English- 
monolingual according to parental reports. They were recruited from preschools in the greater Los Angeles area as well as through a shared-lab database and were compensated with a small gift (e.g. a book). Three children were excluded from the study due to fussiness; they were not included in the final sample of 14 .

Design. Study 1 utilized a within-subjects quasi-experimental design. The comparison variable was children's label-learning experience within the superordinate categories of animals, clothing, and foods, operationally defined as their vocabulary sizes within each category relative to each other. The outcome variable was their ability to learn labels for additional category members, operationally defined as their performance on a generalization test.

Materials. Vocabulary questionnaires. We used the MacArthur-Bates Communicative Development Inventory: Words and Sentences (MCDI; Fenson et al., 1993) to measure children's productive vocabularies. Although the MCDI includes labels for animals, clothing items, and foods, we created an additional vocabulary questionnaire to determine if children comprehended and/or produced labels for members of the superordinate categories that might be included in the generalization test (see Appendix A). The questionnaire also included labels for category members similar to those that might be included in the generalization test (e.g. tortoise was included in the animal category pool; therefore, "tortoise" and "turtle" were included on the vocabulary questionnaire). Parents completed both vocabulary questionnaires prior to the beginning of the study.

Category pools. For each superordinate category, we created a pool of twelve rare members that might be included in the generalization test. A member was considered "rare" if participants were unlikely to produce their labels. For example, both scorpions and dolphins were included in the animal pool because their labels do not appear on the MCDI, which suggests that the typical child does not produce them before they are 30 months of age (Fenson et al., 1993). Goggles and headband were included in the clothing pool, and couscous and tapioca were included in the food pool for the same reason. Based on vocabulary norms, it was possible that participants produced labels for some category members in each of the pools (Wordbank); however, it was unlikely they did so. For example, 10\% of 18-month-olds produce the label for alligator, which was in the animal pool; $10 \%$ of 18 -month-olds produce the label for mittens, which was in the clothing pool. It was likely that children were generally familiar with some of category members in each pool; however, our focus was on children's production of their labels.

For each child, we randomly selected four category members from each pool to include in the generalization test; however, if a child already produced the label of a selected member (as indicated by the MCDI and/or the supplemental vocabulary questionnaire), we randomly selected a different one from the remaining category members in the pool. We did so to prevent children from being tested in their ability to generalize labels that were already in their productive vocabularies. 
Table 1. Category pools used in Studies 1 and 2

\begin{tabular}{ccc}
\hline $\begin{array}{c}\text { Animals } \\
\text { (Studies 1 and 2) }\end{array}$ & $\begin{array}{c}\text { Clothing items } \\
\text { (Studies 1 and 2) }\end{array}$ & $\begin{array}{c}\text { Foods } \\
\text { (Study 1) }\end{array}$ \\
\hline Alligator & Bonnet & Apricots \\
Armadillo & Bootie & Applesauce \\
Butterfly & Barrette & Couscous \\
Dolphin & Ear muffs & Gummies \\
Gopher & Goggles & Lentils \\
Octopus & Headband & Noodles \\
Parrot & Jumper & Pretzels \\
Ram & Mitten & Peanut butter \\
Scorpion & Nose plug & Raisins \\
Seahorse & Sunglasses & Rice \\
Tortoise & Sandals & Tapioca \\
Wolf & Vest & Wheat \\
\hline
\end{tabular}

Object sets. For each category member that was selected to be included in a child's generalization test, we assembled a set of five test objects. As can be seen in Figure 1, object sets included a labeled object, a target object, and three distractor objects. Labeled objects were instances of the category members, which were labeled for children during generalization test trials. The nature of the labeled objects varied between the superordinate categories. For animals, labeled objects were small plastic or wooden figurines with realistic details (e.g. eyes). For clothing items, labeled objects were small but functional items of clothing (e.g. baby or doll clothes). For foods, labeled objects were small portions of actual foods presented on a paper plate.

Target objects were additional instances of the category members; they were considered the correct choices on generalization test trials. They were chosen or created to match the labeled objects in multiple category-relevant perceptual features (for animals and clothing, shape and texture; for foods, material and color) and to mismatch the labeled objects in a perceptual feature that is less relevant to labeling within the category (for animals and clothing, color; for foods, shape). As can be seen in Figure 1, both the labeled object and the target object are alligator figurines with four legs, a tail, and scales; however, the labeled object is a grey and yellow whereas the target object is dark brown. To be concise, we will refer to the perceptual features that are less relevant to labeling within the category as "category-irrelevant", but we acknowledge that they might be relevant to labeling some instances of animals, clothing items, or foods.

Distractor objects were considered incorrect choices on generalization test trials. They were chosen or created to match the labeled objects in shape, color, and superordinate category, respectively. This resulted in each test set having one distractor object that matched the labeled object in a single categoryrelevant feature (for animals and clothing, shape; for foods, material) and that mismatched the labeled object in category-irrelevant perceptual features (for 
animals and clothing, color and/or texture; for foods, material and color), and one distractor object that matched the labeled object in a category-irrelevant feature (for animals and clothing, color; for food, shape) and that mismatched the labeled object in category-relevant features (for animals and clothing items, shape and/ or texture; for foods, material). For example, in Figure 1, the shape-matched object has the same "alligator" shape as the labeled object, but a different color (orange) and texture (bumpy); the color-matched object, being a monkey, has a different shape, but the same color pattern (grey and yellow). The categorymatched object was considered a "super distractor" to make the task sufficiently challenging to children. It mismatched the labeled object in all category-relevant and category-irrelevant perceptual features. In Figure 1, the category-matched object is a starfish. Because of these parameters, and the diversity within the category pools, the object sets varied widely and were made out of a variety of materials. For example, the shape-matched object was the same scorpion figurine covered in purple pipe cleaner; the labeled object for the goggles object set were blue and white children's goggles and the shape-matched object were pieces of pink plastic assembled into a "goggles" shape. Importantly, any distractor objects that realistically represented other members of a category were selected from a pool of additional rare members that was created for distractor objects and were only used in a child's object set if they did not know their labels (according to the MCDI or the supplemental vocabulary questionnaire). Despite the rarity of these additional category members, a few children still knew their labels. When this was the case, a replacement was found within the distractor pool. If this was not possible, a new category member and object set was randomly selected for the child's object set. We took these steps to ensure that children could not use mutual exclusivity to determine the correct referent of the label (Markman, 1991). Thus, a child who experienced the generalization test trial represented by Figure 1 would not have known the labels for monkey or starfish.

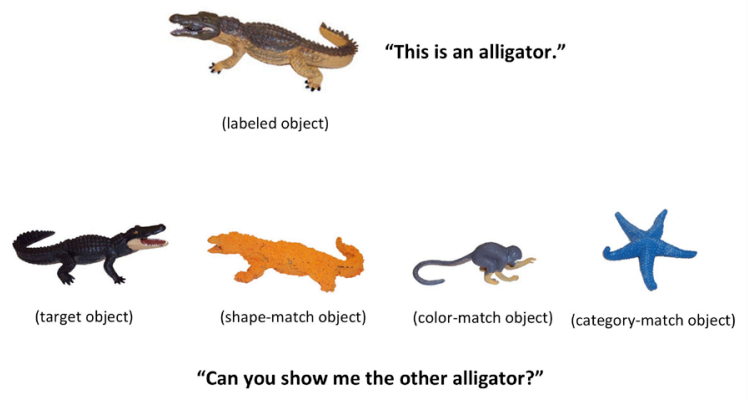

Figure 1. A generalization test trial for Study 1 and 2 with the alligator set. 


\section{Procedure}

The generalization test served to introduce children to the labels of the superordinate category members and test their ability to generalize the labels to new instances of the same members. It was conducted in the laboratory or a quiet corner of children's daycare facilities. Children sat a small table across from the experimenter. If present, parents sat next to their children; they were asked to encourage their children to participate, but to avoid influencing their choices on trials.

The generalization test consisted of twelve trials, one for each of the superordinate category members. Figure 1 depicts the procedure of a single test trial. As can be seen, the experimenter began each trial by presenting children with the labeled object. The experimenter allowed children to play briefly with the object (or if a food, sample it) before placing it in the middle of the table. The experimenter established joint attention with children on the object, pointed to it, and labeled it five times using varied syntactical frames (e.g. "This is a scorpion!"). Experimenters labeled the object five times when joint attention was established with the children. The goal was to give children five good opportunities to pair the word with the object. It was sometimes the case that the label was repeated a few more times in addition to the five, if the child was looking away or spoke over the experimenter.

Next, the experimenter then presented children with the target object and distractor objects, allowing them to play object with each briefly. Finally, the experimenter asked children to select another instance of the superordinate category member from the array (e.g. "Can you give me the other scorpion?"), giving them neutral feedback once they made their selection (e.g. "Thank you!"). Importantly, the superordinate categories themselves were never named during the trials. In other words, labeled objects were never referred to as an "animal", "clothing item", or "food".

\section{Results and discussion}

We analyzed our data in three ways. We first compared children's raw vocabulary sizes between the superordinate categories. Next, we coded their vocabulary sizes within the superordinate categories relative to each other (i.e. small, medium, large). Finally, we examined children's generalization test performance as a product of their raw and relative vocabulary sizes.

Raw and relative vocabulary sizes. We calculated children's raw vocabulary size within each superordinate category by tallying the number of labels for members that they produced as indicated by both the MCDI and additional vocabulary questionnaire. A one-way repeated-measures ANOVA indicated that there were statistically significant differences in children's raw vocabulary sizes between categories, $F(2,26)=3.80, p<0.05$, partial $\eta^{2}=0.32$. Post-hoc analyses indicated that children knew significantly more labels for animals $(M=12.58$, 
$S D=15.16)$ than for clothing items $(M=5.00, S D=6.85), L S D<0.05$. There were marginally statistically significant differences in the number of labels children knew for clothing items $(\mathrm{M}=5.00, \mathrm{SD}=6.85)$ and for foods $(M=9.42, S D=13.06), L S D<0.10$. There were no statistically significant differences between children's animal and food vocabulary sizes.

These between-category differences in raw vocabulary sizes were consistent with those observed in previous work (e.g. Gershkoff-Stowe \& Smith, 2004) as well as with vocabulary norms (Fenson et al., 1993). Recall that in Study 1 we treated children's raw vocabulary sizes in the superordinate categories as proxy measures of their experience in learning labels for members. Thus, our findings suggest that, as a group, children had more experience learning labels for animals than for clothing items or foods. However, there was variability in the "ranking" of individual children's vocabulary sizes within the superordinate categories: Although many children knew the most labels for animals, and fewer labels for clothing items and foods, not all followed this trend. Because our predictions concerned the amount of children's label-learning experience within the superordinate categories, rather than in which superordinate categories they had the most (or least) label-learning experience, we coded children's raw vocabulary sizes relative to each other (e.g. small, medium, and large). For example, if a child knew 8 labels for animals, 4 labels for clothing items, and 11 labels for foods, their raw vocabulary sizes would be coded as medium, small, and large, respectively. On average, children knew 3.85 labels $(S D=6.36)$ for members of the superordinate category within which they had a small relative vocabulary size. Children knew 7.93 labels $(S D=11.74)$ for members of the superordinate category within which they had a medium relative vocabulary size and 11.93 labels $(S D=14.69)$ for members of the superordinate category within which they had a large relative vocabulary size.

Generalization test performance. We hypothesized that children would correctly generalize more labels for members of the superordinate categories within which they had more experience than for members of the superordinate categories within which they had less label-learning experience. To test this hypothesis, we compared the proportion of test trials that children chose target objects between the superordinate categories within which they had small, medium, and large relative vocabulary sizes, respectively (see Figure 2). A one-way repeated-measures ANOVA revealed statistically significant between-category differences in the frequency of children's target object choices, $F(2,26)=3.71, p<0.05$, partial $\eta^{2}=0.22$. Post-hoc analyses indicated that children chose target objects more frequently on test trials for the category within which they had a large relative vocabulary size $(M=0.66, S D=0.06)$ than on test trials for the category in which they had a medium relative vocabulary size $(M=0.48, S D=0.05), L S D<0.05$. There were marginally statistically significant differences in the frequency of children's target object choices between test trials for the categories within which they had a large relative vocabulary size and 
a small relative vocabulary size $(M=0.52, S D=0.07), L S D<0.10$. There were no statistically significant differences in the frequency of children's target object choices between test trials for the categories within which they had a small relative vocabulary size and a medium relative vocabulary size. Additionally, a series of one-sample t-tests indicated that children chose target objects more frequently than would be expected by chance $(0.25)$ on test trials for the category within which they had a small relative vocabulary size, $t(13)=3.74, p<0.01$ (two-tailed), Cohen's $d=1.00$. They also chose the target objects with abovechance frequency on test trials for the categories within which they had a medium relative vocabulary size, $t(13)=4.76, p<0.05$ (two-tailed), Cohen's $d=1.27$, and a large relative vocabulary size, $t(13)=7.30, p<0.01$ (two-tailed), Cohen's $d=1.95$.

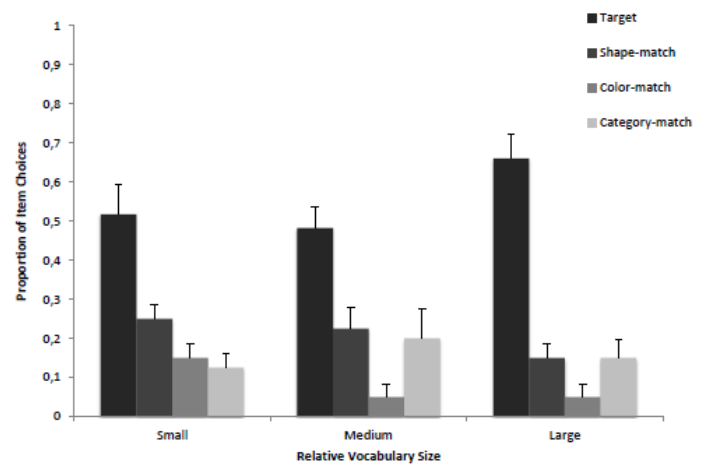

Figure 2. Mean proportion of children's object choices as a product of their relative vocabulary size in Study 1 . Bars represent Standard Error.

Between superordinate categories. Given the group trends in children's raw vocabulary sizes, it was possible that generalizing labels for animals was somehow easier for children than generalizing labels for clothing items or foods for reasons other than the extent of their label-learning experience (e.g. animals might be more salient to children, and thus, the perceptual features relevant to their categorization labeling are more easily discernible). To rule out this possibility, we ran a one-way repeated-measures ANOVA comparing the proportion of test trials that children chose target objects between superordinate categories. No statistically significant effects were indicated. Children chose target objects with comparable frequency on animal, clothing, and food test trials. Additionally, a series of one-sample t-tests indicated that children chose target objects more frequently than would be expected by chance $(0.25)$ on animals test trials, $t(13)=$ 5.93, $p<0.01$ (two-tailed), Cohen's $d=1.68$. They also chose target objects with above-chance frequency on clothing test trials, $t(13)=3.63, p<0.05$ (two-tailed), Cohen's $d=1.05$, and food test trials, $t(13)=4.17, p<0.01$ (two-tailed), Cohen's $d=1.21$. Thus, it was unlikely that children's generalization test performance did not vary due to differences in the "learnability" of labels for animals, clothing items, or foods. 
Taken together, these findings were consistent with our hypothesis. Children properly generalized labels for members of all superordinate categories, which was to be expected based on the fact they had some experience in learning labels for members of each superordinate category. However, they properly generalized more labels for members of the superordinate category within which they had more label-learning experience than for members of the superordinate categories within which they had less label-learning experience; this pattern held true regardless of the superordinate categories within which they had more or less label-learning experience.

Correlations. Finally, we ran a series of Pearson product-moment correlations exploring the relationship between children's raw vocabulary sizes and their overall productive vocabulary size to their performance on the generalization test. No statistically significant effects were indicated; children's raw and overall vocabulary sizes did not predict their performance on the generalization test. These findings were somewhat surprising in light of the findings of previous research (e.g. Bates et al., 1988; Jones \& Smith, 2002; Gershkoff-Stowe \& Smith, 2004; Samuelson \& Smith, 1999 but cf. Tan \& Schafer, 2005; Thom \& Sandhofer, 2009), but were not necessarily inconsistent with our predictions. We address their implications in the General Discussion.

\section{Study 2}

Study 1 provided evidence that children's label-learning experience within a superordinate category from their every day lives predicts their ability to learn labels for additional members; however, its quasi-experimental design ultimately prevented us from determining the direction of this relationship. A plausible interpretation of our findings was that differences in children's proficiency in label learning within the superordinate categories led to differences in their raw and relative vocabulary sizes (rather than the reverse, as we hypothesized). In Study 2, we sought to rule out this possibility by experimentally manipulating children's label-learning experience within the superordinate categories of animals and clothing and testing their ability to generalize labels for additional members of each superordinate category.

\section{Method}

Participants. Seventeen children (eight males) participated in Study 2. On average, they were 23.79 months $(S D=2.73$, range $=20.10-29.00)$ of age and had 233.00 words $(S D=161.82$, range $=29-607)$ in their overall productive vocabularies. We recruited slightly older children for Study 2 to increase the likelihood of their cooperation over multiple training and testing sessions. All children were English-monolingual according to parental reports. They were recruited from preschools in the Los Angeles area and compensated with a small gift. Nine children were randomly assigned to the animal greater training group; 
eight children were randomly assigned to the clothing greater training group. An additional five children were excluded due to failure to cooperate and two were excluded due to experimenter error; they were not included in the final sample of 17.

Design. Study 2 utilized a mixed between- and within-subjects experimental design. The between-subjects variable was children's training group: In the animal greater group, children were trained in the labels for six rare members of the superordinate category of animals and for two rare members of the superordinate category of clothing; in the clothing greater group, children were trained in the labels for six rare members of the superordinate category of clothing and for two rare members of the superordinate category of animals. The within-subjects variable was the number of labels for members of the superordinate categories in which children had been trained (i.e. six vs. two). The dependent variable was children's ability to learn labels for additional category members, operationally defined as their performance on a generaliz ation test.

Materials. As in Study 1, we used the MCDI to measure children's overall productive vocabularies. We used a supplementary questionnaire to measure children's productive vocabularies in the superordinate categories; it was similar to the one used in Study 1, but did not include labels for foods. Parents completed both questionnaires prior to the beginning of the study.

Category pools. Study 2 used the animal and clothing category pools from Study 1 . For children in the animal greater group, we randomly selected six animals and two clothing items from the category pools to use in training. For children in the clothing greater group, we randomly selected six clothing items and two animals from the category pools to use in training. For each child, regardless of his or her training condition, we randomly selected four members from each category pool to use in testing; however, we replaced any category members for which children already knew the labels (as indicated by the vocabulary questionnaires) with a new random selection. Thus, we never tested children's ability to generalize labels they already produced.

Object sets. The animal and clothing item object sets used in Study 1 were also used in both training and testing in Study 2. In Study 2, training utilized only some of the objects from the sets: The labeled objects and target objects served as multiple instances of category member and the category-matched object served as a foil. Testing utilized all objects from the sets (i.e. the labeled object, the target objects, and all distractor objects). As in Study 1, any objects that realistically represented other members of a category were selected from a pool of additional rare members that was created for distractor objects and were only used in a child's object set if they did not know their labels (according to the MCDI or the supplemental vocabulary questionnaire). If children knew their labels, a replacement was found within the distractor pool. If this was not possible, a new category member, and object set, was randomly selected for the child's object set. 
Procedure. Study 2 consisted of two sessions conducted on separate days. Training took place during Sessions 1 and 2; testing took place during Session 2. Both sessions were conducted in a quiet corner in the children's daycare facility where, in most cases, children sat at a small table across from the experimenter. Due to variability in the children's attendance schedule, Sessions 1 and 2 were not always conducted on consecutive days, but were always completed within the span of a week.

Training. A training session consisted of eight trials, one for each category member. The order of the trials was randomized between sessions. As can be seen in Figure 3, the experimenter began each trial by presenting children with an instance of the category member (i.e. the labeled object from the set). The experimenter allowed children to play briefly with the object before placing it in the middle of the table. The experimenter established joint attention with children on the object, pointed to it, and labeled it approximately five times using varied syntactical frames (e.g. "This is a scorpion!" or "Look: A scorpion!"). Next, the experiment presented children with a second instance of the category member (i.e. the target object from the set). Again, the experimenter established joint attention with children on the object, pointed to it, and labeled it approximately five times using varied syntactical frames (e.g. "This is also a scorpion!" or, "There are two scorpions!"). The experimenter presented children with the foil object (i.e. the category-matched distractor object from the set) and alerted them to the fact that it was not an instance of the category member, but did not label it (e.g. "This is not a scorpion. Let's put it away."). The foil object was included to allow children to determine the specific perceptual features of the labeled objects that were relevant for their categorization and labeling. The experimenter then removed the foil from sight, leaving the two labeled category members on the table.

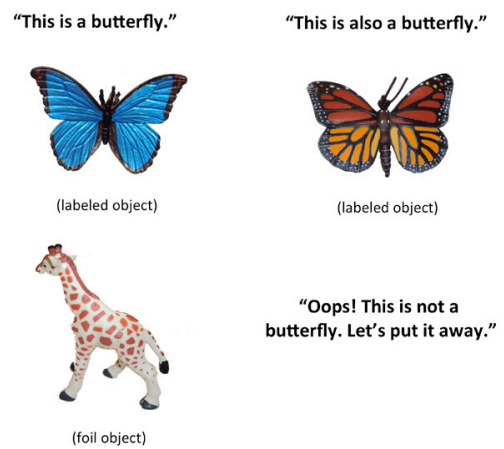

Figure 3. Study 2 training procedure with butterfly training set. The set includes two labeled instances of the category member (the blue butterfly and orange butterfly) and a foil object (the giraffe).

Testing. The generalization test procedure in Study 2 was identical to that in Study 1. However, it consisted of only eight trials in total, one for each of the to-be-tested category members. 


\section{Results and discussion}

We analyzed data from Study 2 in three ways. We first compared participant and experimental characteristics between training groups. Next, we examined children's generalization test performance within each superordinate category as a product of the number of labels for members in which they had been trained. Finally, we compared children's generalization test performance between superordinate categories.

Participant and experimental characteristics. A series of independentsample t-tests indicated that there were no statistically significant differences in children's age, overall vocabulary size, and raw clothing and animal vocabulary sizes, or experimental span between the animal-greater and clothing-greater groups. Thus, participant characteristics and experimental characteristics were comparable between experimental conditions. However, a series of dependentsamples t-tests indicated that, across training groups, children knew significantly more labels for animals $(M=25.35, S D=3.39)$ than for clothing items $(M=14.18, S D=2.06)$ at the outset of the study, $t(16)=5.07, p<0.05$ (twotailed), Cohen's $d=1.23$. These between-category differences in vocabulary sizes were consistent with those observed in Study 1 and with vocabulary development norms (Fenson et al., 1993). The fact that children had vocabularies of any size within the superordinate categories of animals and clothing suggested that they had at least some previous experience in learning labels for members of each category. Thus, it is important to clarify that in Study 2 we manipulated their label-learning experience beyond what they had already had in their daily lives. However, it is important to note that not all children followed group trends: There were children in both training groups that knew more labels for clothing items than for animals. The between-category differences in children's vocabulary sizes meant that some children in the clothing-greater training group may have had less of a differential in their total amount of experience (from their daily lives and training) in learning labels for animals and clothing items than children in the animal greater training group. We address the implications of this possibility in the General Discussion.

Generalization test performance. We hypothesized that children would be better able to learn labels for members of categories within which they have had more label-learning experience than labels for members of categories within which they have had less label-learning experience. To test this hypothesis, we collapsed across training groups, and compared the proportion of test trials that children chose target objects in the superordinate categories within which they were trained in the labels for two and in the labels of six members. As can be seen in Figure 4, children's performance on the generalization test appears to have varied between superordinate categories as a product of the number of labels in which they were trained. A dependent-samples t-test confirmed that children chose target objects more frequently on test trials for the category within which they were trained in the labels of six members $(M=0.55 ; S D=0.24)$ than on 
test trials for the category within which they were trained in the labels of two members $(M=0.39 ; S D=0.27), t(16)=2.10, p=0.05$ (two-tailed), Cohen's $d=0.51$. Additionally, a series of one-sample t-tests indicated that children chose target objects more frequently than what would be expected by chance $(0.25)$ on the test trials for the category within which they were trained in the labels for two members, $t(16)=2.28, p<0.05$ (two-tailed) Cohen's $d=0.55$. They also chose target objects with above-chance frequency on test trials for the category within which they were trained in the labels for six members, $t(16)=5.25, p<0.01$ (two-tailed), Cohen's $d=1.29$.

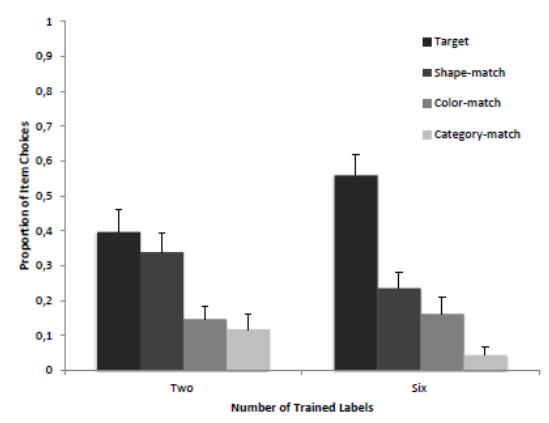

Figure 4. Mean proportion of children's object choices in Study 2 as a product of the number of labels for category members in which they were trained. Bars represent Standard Error.

Given the group trends in children's raw vocabulary sizes, it was possible that the between-category differences in children's generalization test performance was primarily driven by children in the animal-greater group-because the differences in their label-learning experience between the superordinate categories were potentially more extreme than those of the clothing-greater group, or more simply, because they were already better able to generalize labels for animals than for clothing items. To explore this possibility, we compared the proportion of test trials that children chose target objects between superordinate categories and training groups (see Figure 5). A mixed between- and within-subjects ANOVA indicated a marginally statistically significant interactive effect of category and training group on the frequency of children's target object choices, $F(1,15)=4.10$, $p=0.06$, partial $\eta^{2}=0.21$. Children in the animal greater group tended to choose target objects more frequently on animal test trials than on clothing test trials, whereas children in the clothing greater group tended to choose target objects more frequently on clothing test trials than on animal test trials. Thus, the pattern of results that we observed across superordinate categories appears to have held true within each superordinate category. In other words, even the children who knew more labels for animals at the outset of the study appear to have properly generalized more labels for clothing items than for animals in the generalization test. This suggests that training had an effect on children's ability to generalize labels properly above and beyond that of their label-learning experience in their 
daily lives and implies that label-learning experience in the laboratory might somehow differ from label-learning experience in the real world; we address this implication in the General Discussion.

Between categories. A dependent-samples t-test indicated that, across training groups, there were no statistically significant differences in children's generalization test performance between the superordinate categories. Children

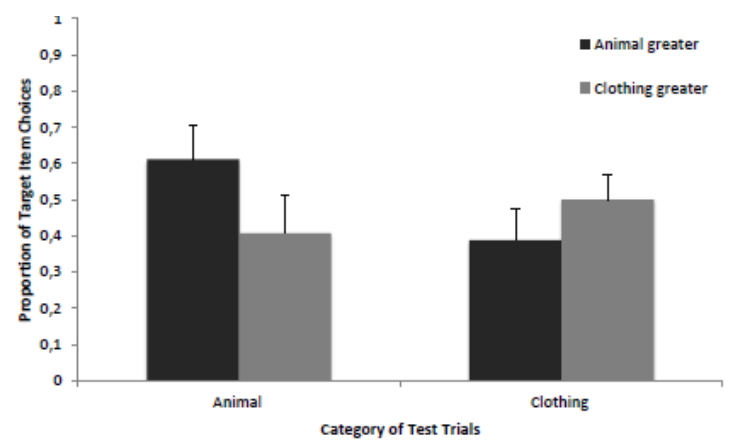

Figure 5. Mean proportion of children's choice of target objects on animal and clothing test trials as a function of their training group. Bars represent Standard Error.

chose target objects with comparable frequency on animal test trials and clothing test trials. Additionally, a series of one-sample t-tests indicated that children chose target objects more frequently than would be expected by chance $(0.25)$ on animals test trials, $t(16)=3.65, p<0.01$ (two-tailed), Cohen's $d=0.90$, and on clothing test trials, $t(16)=3.50, p<0.05$ (two-tailed), Cohen's $d=0.83$. This provided further support to the conclusion that children's performance on the generalization test-although strong overall-varied between superordinate categories as a product of the number of labels in which they were trained.

Taken together, these findings were consistent with our hypothesis. With participant and experimental characteristics comparable between training groups, children properly generalized more labels for members of the superordinate category within which they had more label-learning experience than for members of the superordinate categories within which they had less label-learning experience; this pattern held true regardless of their training group. These findings expand on the findings of previous research (e.g. Smith et al., 2002; Thom \& Sandhofer, 2009), and bolster those of Study 1 by suggesting that the relationship between children's label-learning experience and label-learning ability is category-specific and causal in nature.

Correlations. Finally, we ran a series of Pearson product-moment correlations exploring the relationship between children's raw vocabulary sizes and their overall productive vocabulary size and their performance on the generalization test. As in Study 1, no statistically significant effects were indicated; children's raw and overall vocabulary sizes did not predict their performance on the generalization test. Again, these findings were somewhat surprising, but not 
necessarily inconsistent with our predictions; we address their implications in the General Discussion.

\section{General Discussion}

In two studies, we documented the relationship between children's labellearning experience and their label-learning ability within superordinate categories with diverse perceptual organization. We found that, although children performed well on all generalization test trials, they performed better on those for the superordinate categories within which they knew more labels or had been trained in more labels. This suggests that children's label-learning experience within one superordinate category facilitated their learning of labels for additional members of that category-but only of that category. These findings were consistent with our general prediction that children's category-specific labellearning strategies develop and function independently with each superordinate category. Furthermore, recall that selecting the correct answers (i.e. the target objects) on generalization test trials required children to map and generalize the category members' labels by all the perceptual features relevant to their labeling, which differed between categories (e.g. shape, texture, and the presence of eyes for animals; color and material for foods). Thus, our findings suggest that children were utilizing multiple strategies for determining the features of the labeled object that were relevant to their labeling-and that their effectiveness in doing so depended on their label-learning experience within each superordinate category.

Our overarching objective was to better understand the factors that facilitate children's label learning within superordinate categories with diverse perceptual organization. Our findings suggest that children's label-learning experience promotes their label- learning ability within superordinate categories just as it does across them (Gershkoff-Stowe \& Hahn, 2007; Gershkoff-Stowe \& Smith, 2004; Mervis \& Bertrand, 1994/1995; Smith et al., 2002; Thom \& Sandhofer, 2009). In doing so, they provide a possible explanation for the betweencategory differences in children's mapping and generalization behavior that have been observed in previous work (e.g. Lavin \& Hall, 2001). For example, our findings suggest that, by three years of age, children in Lavin and Hall (2001) had developed both a "toy bias" and a "food bias" from their experience in learning labels for members of each superordinate category; these biases were activated when the categories were cued during generalization test trials, causing children to apply labels to the novel objects accordingly. Furthermore, children had developed these strategies not as the result of their age itself, but the labellearning experience they had accumulated by that age.

\section{Support for general predictions}

We made a number of other general predictions regarding the nature of 
category-specific label-learning strategies. First, we predicted that such strategies take the form of attentional biases: Children's attention is automatically drawn to the perceptual features of a labeled object that have been historically relevant to labeling members of its superordinate category, making them more likely to map and generalize its label properly. Our findings were consistent with this prediction in that children's generalization test performance varied between superordinate categories despite the fact that categories were cued neither explicitly (e.g. by referring to foods as "something to eat" as in Lavin and Hall, 2001) nor implicitly (e.g. by organizing trials in category blocks). This suggests that the perceptual features of the labeled objects alone were sufficient to activate children's attentional biases, and thereby facilitate their label learning (Smith, 2000/2001; Jones \& Smith, 1998/2002). This does not rule out the possibility that children's category-specific label-learning strategies were functioning in a "topdown" manner; however, it leaves open the possibility that they were functioning in the absence of full-fledged ontological understanding of the labeled objects.

We also predicted that children develop category-specific attentional biases because they are exposed to the perceptual regularities among members of superordinate categories via their label-learning experience. It is therefore significant that we found evidence for the relationship between children's labellearning experience and label-learning ability within real, and really common, superordinate categories. It is likely that the children in Studies 1 and 2 encounter members of the superordinate categories of animals, clothing items, and foods on a daily basis. If simple exposure to members of superordinate categories promoted the development of category-specific label-learning strategies, we would expect children's performance to have been comparable in all test trials; instead, their performance varied as a product of their vocabulary sizes or the number of labels in which they were trained within each category. Thus, our findings suggest that the experience of learning labels for members of superordinate categories uniquely promotes the development of categoryspecific label-learning strategies-perhaps because applying the same labels to members of a basic-level category encourages children to see the perceptual features common among them, and to make generalizations about the relevance of those features to labeling across the superordinate category in which they are nested (Jones \& Smith, 2002; Smith, 2000/2001; Smith et al., 2002).

An additional benefit of using real superordinate categories was that it allowed us to determine the extent of children's sensitivity to the perceptual regularities among members. Unlike tightly controlled experimenter-created categories, real superordinate categories have imperfect perceptual organization (Russell \& Kyger, under review). Thus, an implication of our findings within the current theoretical framework is that children's attention can "tune" to the perceptual features that are collectively relevant to labeling within a superordinate category even if those features are not shared by all members. Put simply: Children can make clean generalizations based on messy data. 
A drawback of using real superordinate categories was that it prevented us from controlling children's previous experience in learning labels for animals and clothing items in Study 2. Indeed, children's animal and clothing item vocabulary sizes suggest that they had already amassed at least some labellearning experience within both superordinate categories prior to the start of the study. It is possible that children's generalization test performance varied between superordinate categories as a product of the label-learning experience they had gained both inside and outside of the laboratory. For this reason, we cannot conclude that in Study 2 we created children's category-specific labellearning strategies via training; however, we can reasonably conclude that we accelerated their development.

Finally, we predicted that attentional biases could develop within any superordinate category regardless of its perceptual organization. Our findings were consistent with this prediction in that we found the same pattern of results regardless of the superordinate categories within which children had more or less label-learning experience. For example, the children in Study 2 who were trained in more labels for animals than for clothing items properly generalized more labels for animals than for clothing items; conversely, children who were trained in more labels for clothing items than for animals properly generalized more labels for clothing items than for animals. This suggests that the perceptual organization of the superordinate category matters less than children's exposure to the regularities among its members via their linguistic experience. Furthermore, they suggest that category-general label-learning strategies do not hinder the development of category-specific label-learning strategies. For example, even our younger participants in Study 1, who based on their age should be experiencing the peak of the shape bias, generalized the labels of foods by material. These findings in particular position our account as a viable explanation for how children develop label-learning proficiency within all superordinate categories-even those for which shape does not matter, or that are inanimate, and so forth.

Alternative interpretations. We have articulated reasons why our findings uniquely support our general predictions; however, our findings can also be explained in other ways. For example, it is possible that children's label-learning strategies were rooted in their ontological understanding of the superordinate categories of animals, clothing items, and foods, which they gained through their experience learning labels for members. The more label-learning experience they had within a superordinate category, the more they knew about its members (or about labeling its members), and the more they properly mapped and generalized labels for additional members. If this interpretation were accurate, our findings would carry a number of implications for this theoretical framework. First, it would suggest that the act of labeling objects somehow functions to promote children's ontological understanding of the superordinate category to which they belong. It is possible that children's ontological understanding facilitated their label learning independent of their label-learning experience. For example, children 
who simply knew more about animals were better able to choose new instances of animals during generalization test trials. However, this would not explain our findings from Study 2, where generalization test performance was boosted from label training, or why children do not develop the shape bias until after they have amassed sizeable shape vocabularies-even though they are regularly exposed to objects from shape-based categories. It would also be inconsistent with research suggesting that labeling creates "essence" placeholders, encouraging children to look for deeper commonalities (Gelman \& Coley, 1991; Gelman et al., 1994). Within this theoretical framework, our findings also raise the question of how children's ontological understanding is activated in the absence of cues to the superordinate category of a labeled object. It has been proposed that children get better at detecting category-relevant features in the absence of cues over time (Booth \& Waxman, 2002b); our findings suggest that this occurs because they gain label-learning experience.

Our design and methodology prevent us from determining which interpretation of our findings is accurate-but, again, this was not our objective. At the heart of our predictions, and those of this alternative theoretical framework, is the idea is that children build an arsenal of category-specific label-learning strategies via their label-learning experience. Our findings provide strong evidence for this phenomenon, even if the nature of the strategies remains up for debate.

\section{Additional contributions}

A number of methodological choices further strengthened the contribution of our findings. For example, in Study 1 we chose to measure children's labellearning experience using their relative vocabulary size (based on MCDI measurements). Jones and Smith (2002) found that children with larger noun vocabularies exhibited differential mapping and generalization behavior between the superordinate category of animals and the class of artifacts. The underlying assumption was that the more nouns children knew, the more labels for animals and artifacts they also knew. However, the researchers did not measure the sizes of children's animal and artifact vocabularies specifically. Thus, our findings supplement theirs by providing direct evidence for the relationship between label-learning experience and label-learning ability within the superordinate categories of animals-as well as within the superordinate categories of clothing items and foods.

In Studies 1 and 2, we chose to recruit younger samples compared to those of similar studies (e.g. Jones \& Smith, 2002; Lavin \& Hall, 2001). The children in our Studies 1 and 2 were 18 and 24 months, respectively, whereas the children in Jones and Smith (2002; experiments 1 and 2) and Lavin and Hall (2001) were closer to two and a half and three years of age. Because of these differences, our findings appear to stand in contrast to theirs, which suggest that children do not effectively utilize category-specific label-learning strategies until they are somewhat older. We have argued that, in the development of label-learning 
proficiency, label-learning experience matters more than age. Our findings with younger children speak to this point. Even by their second birthdays, the children in Studies 1 and 2 had accumulated at least some label-learning experience within each superordinate category. They had the opportunity to begin developing labellearning strategies within them. However, the fact that children's generalization test performance varied between superordinate categories suggests that their efficacy in utilizing those strategies also varied. Thus, unlike previous work, our studies appear to have captured a moment in time when children have not yet developed label-learning proficiency in all superordinate categories to the same degree.

Furthermore, in Study 2 specifically, we designed training to increase the quality of children's label-learning experience within the superordinate categories in addition to its quantity. For example, we provided children with multiple cues to the perceptual features that were (and were not) relevant labeling within the superordinate categories; this is a common methodological choice in label training studies (e.g. Jones \& Smith, 2002; Thom \& Sandhofer, 2009) because simultaneously comparing and contrasting instances aids in category generalization and concept abstraction (Gentner \& Markman, 1994; Gentner \& Namy, 1999; Namy \& Gentner, 2002). Additionally, by administering all training trials on a single day, we made children's label-learning experience within the superordinate categories much more concentrated than it would have been in their daily lives. For these reasons, children in Study 2 might have developed categoryspecific label-learning strategies more quickly than children in previous work (e.g. Jones \& Smith, 2002; experiments 1 and 2; Lavin \& Hall, 2001), who did not receive training. Consistent with this interpretation is the fact that Jones and Smith (2002; experiment 3 ) found that, with training in animal-like experimentercreated categories, 22-month-old children begin to exhibit an animal-specific label-learning strategy (e.g. an apparent shape-and-texture attentional bias). Thus, our findings also hint at the optimal conditions for developing categoryspecific label-learning strategies. The amount of label-learning experience within each superordinate category need not be vast-exposure to a handful of labels for members will suffice (Jones \& Smith, 2002; Thom \& Sandhofer, 2009)-as long as it is rich in information.

\section{Additional considerations}

Vocabulary sizes. Given the results of similar studies (e.g. Gershkoff-Stowe \& Smith, 2004; Mervis \& Bertrand, 1994; Samuelson \& Smith, 1999; Woodward et al., 1994 but cf. Tan \& Schaffer, 2005; Thom \& Sandhofer, 2009), it was somewhat surprising that, in both Studies 1 and 2, children's raw vocabulary sizes within each superordinate category did not predict their performance on the corresponding generalization test trials. However, this previous work focused on, and found support for, the relationship between children's label-learning ability and their overall or total noun vocabulary sizes; these latter variables 
exist on a much broader scale than children's vocabulary sizes within individual superordinate categories. It could be the case that our findings (or lack thereof) resulted from the inability of our relatively brief vocabulary questionnaire to capture subtle variability of children's label-learning experience within superordinate categories. Thus, future studies in this same vein should consider using more thorough measures of children's label-learning experience within various superordinate categories.

However, it could also be the case that the development of children's labellearning biases does not directly depend on their mastery of the labels in which they have been trained, but rather on their exposure to the perceptual regularities among members of object categories or superordinate categories, which increases with every new label that they learn-or attempt to learn. It is this exposure that encourages them to make generalizations regarding the perceptual features relevant to labeling, tunes their attention to those features, and facilitates their subsequent label learning. Through their label-learning experience, children increase their vocabulary sizes as well as their label-learning ability; these two processes might occur in tandem, but might not always predict each other. By this logic, the differences in children's label-learning ability observed in Study 1 resulted from relative differences in the extent of their label-learning experience, only approximated by their raw vocabulary sizes. The differences in children's label-learning ability observed in Study 2 resulted from relative differences in the extent of their label-learning experience, experimentally manipulated via training. Consistent with this possibility are the findings of Thom and Sandhofer (2009). In their color-label training study, children's generalization test performance increased as a product of the number of color labels in which they had been trained, regardless of whether or not they learned the trained color labels.

Sample sizes. Finally, it should be acknowledged that our sample sizes were relatively small. Although they were comparable to those of the other training studies that inspired out work (e.g. Smith et al., 2002; Thom \& Sandhofer, 2009), this aspect of our methodology should be considered when interpreting the contribution of our findings. Just as this previous work has incrementally added to our understanding of how children develop label-learning proficiency, so too does ours. The current studies serve as an important first step investigating our predictions.

\section{Summary}

We sought to understand better the factors that facilitate children's label learning within superordinate categories with diverse perceptual organization. Our findings suggest that children develop multiple strategies for determining how labels refer to objects, each specific to a superordinate category, through their experience in learning labels for members. They bolster the findings of previous work and carry implications for multiple theoretical frameworks 
of children's label learning. Finally, they prompt future research to explore the root of the relationship between children's label-learning experience and their label-learning ability within superordinate categories.

\section{Acknowledgements}

The author would like to thank Dr. Catherine Sandhofer for her help in completing this project .

\section{References}

Bates, E., Bretherton, I., \& Snyder, L. (1988). From first words to grammar: Individual differences and dissociable mechanisms. New York, NY: Cambridge University Press.

Bloom, L. (1973). One word at a time: The use of single-word utterances before syntax. The Hague: Mouton.

Bloom, P. (2000). How children learn the meanings of words. Cambridge, MA: The MIT Press.

Bloom, P., \& Markson, L. (1998). Intention and analogy in children's naming of pictorial representations. Psychological Science, 9(3), 200-204. doi:10.1111/1467-9280.00038

Booth, A. E., \& Waxman, S.R. (2002). Word learning is "smart": Evidence that conceptual information affects preschoolers' extension of novel words. Cognition, 84(1), B11-B22.

Booth, A. E., Waxman, S. R., \& Huang, Y. T. (2005). Conceptual information permeateswordlearningininfancy.DevelopmentalPsychology,41(3),491-505. doi:10.1037/0012-1649.41.3.491

Bowerman, M. (1978). The acquisition of word meaning: An investigation into some current conflicts. In N. Waterson, C. Snow (Eds.), The development of communication (pp. 263-287). New York, NY: Wiley.

Carey, S. (1978). The Child as a Word Learner. In J. Bresnan, G. Miller, M. Halle (Eds.), Linguistic Theory and Psychological Reality. Cambridge, MA: MIT Press, 264-293.

Carey, S., \& Bartlett, E. (1978). Acquiring a single new word. Proceedings of the Stanford Child Language Conference, 15, 17-29.

Clark, E. V. (1993). The lexicon in acquisition. Cambridge: Cambridge University Press.

Diesendruck, G., \& Bloom, P. (2003). How specific is the shape bias? Child Development, 74(1), 168-178. doi:10.1111/1467-8624.00528

Diesendruck, G., Markson, L., \& Bloom, P. (2003). Children's reliance on creator's intent in extending names for artifacts. Psychological Science, 14(2), 164-168. doi:10.1111/1467-9280.t01-1-01436

Fenson, L., Dale, P. S., Reznick, J. S., Thal, D., Bates, E., Hartung, J. P., Pethick, S., \& Reilly, J.S. (1993). The MacArthur Communicative Development 
Inventories: User's Guide and Technical Manual. Baltimore, MD: Paul H. Brookes Publishing Co.

Gelman, S. A. (1988). Children's expectations concerning natural kind categories. Human Development, 31(1), 28-34. doi:10.1159/000273201

Gelman, S. A., \& Coley, J. D. (1991). Language and categorization: The acquisition of natural kind terms. In S. A. Gelman, J. P. Byrnes, S. A. Gelman, J. P. Byrnes (Eds.), Perspectives on language and thought: Interrelations in development (pp. 146-196). New York, NY: Cambridge University Press. doi:10.1017/CBO9780511983689.006

Gelman, S. A., Coley, J. D., \& Gottfried, G. M. (1994). Essentialist beliefs in children: The acquisition of concepts and theories. In L. A. Hirschfeld, S. A. Gelman, L. A. Hirschfeld, S. A. Gelman (Eds.), Mapping the mind: Domain specificity in cognition and culture (pp. 341-365). New York, NY, US: Cambridge University Press. doi:10.1017/CBO9780511752902.014

Gentner, D., \& Markman, A. B. (1994). Structural alignment in comparison: No difference without similarity. Psychological Science, 5, 152-158. doi: 10.1111/j.1467-9280.1994. tb00652.x

Gentner, D., \& Namy, L. L. (1999). Comparison in the development of categories. Cognitive Development, 14, 487-513. doi:10.1016/S0885-2014(99)00016-7 Gershkoff-Stowe, L., \& Hahn, E. R. (2007). Fast mapping skills in the developing lexicon. Journal of Speech, Language, and Hearing Research, 50(3), 682-696. doi:10.1044/1092-4388(2007/048)

Gershkoff-Stowe, L. \& Smith L. B. (2004). Shape and the first hundred nouns. Child Development, 75, 1098-1114.

Graham, S. A., \& Poulin-Dubois, D. (1999). Infants' reliance on shape to generalize novel labels to animate and inanimate objects. Journal of Child Language, 26(2), 295-320. doi:10.1017/S0305000999003815

Hupp, J. M. (2015). Development of the shape bias during the second year. The Journal of Genetic Psychology: Research and Theory on Human Development, 176(2), 82-92. doi:10.1080/00221325.2015.1006563

Imai, M., \& Gentner, D. (1997). A cross-linguistic study of early word meaning: Universal ontology and linguistic influence. Cognition, 62, 169-200.

Imai, M., Gentner, D., \& Uchida, N. (1994). Children's theories of word meaning: The role of shape similarity in early acquisition. Cognitive Development, 9, $45-75$.

Jones, S. S., \& Smith, L. B. (1998). How children name objects with shoes. Cognitive Development, 13, 323-334.

Jones, S. S., \& Smith, L. B. (2002). How children know the relevant properties for generalizing object names. Developmental Science, 5, 219-232.

Jones, S. S., Smith, L. B., \& Landau, B. (1991). Object properties and knowledge in early lexical learning. Child Development, 62, 499-516.

Keil, F. C. (1994). The birth and nurturance of concepts by domains: The origins of concepts of living things. In L. A. Hirschfeld, S. A. Gelman, L. A. 
Hirschfeld, S. A. Gelman (Eds.), Mapping the mind: Domain specificity in cognition and culture (pp. 234-254). New York, NY: Cambridge University Press. doi:10.1017/CBO9780511752902.010

Kemler Nelson, D. G. (1995). Principle-based inferences in young children's categorization: Revisiting the impact of function on the naming of artifacts. Cognitive Development, 10, 347-380.

Landau, K.B., Smith, L.B., \& Jones, S.S. (1988). The importance of shape in early lexical learning. Cognitive Development, 3, 299-321.

Lavin, T. A., \& Hall, D. (2002). Domain effects in lexical development: Learning words for foods and toys. Cognitive Development, 16(4), 929-950. doi:10.1016/S0885-2014(02)00070-9

Macario, J.F. (1991). Young children's use of color in classification: foods and canonically colored objects. Cognitive Development, 6, 17-46.

Mandler, J. M., \& McDonough, L. (1998). On developing a knowledge base in infancy. Developmental Psychology, 34(6), 1274-1288. doi:10.1037/00121649.34.6.1274

Markman, E. (1989). Categorization and naming in children (pp. 19- 38). Cambridge, MA: MIT Press.

Markman, E. M. (1991). The whole-object, taxonomic, and mutual exclusivity assumptions as initial constraints on word meanings. In S. A. Gelman, J. P. Byrnes, S. A. Gelman, J. P. Byrnes (Eds.), Perspectives on Language and Thought: Interrelations in Development (pp. 72-106). New York, NY: Cambridge University Press.

Mervis, C.B., \& Bertrand, J. (1995). Early lexical acquisition and the vocabulary spurt: A response to Goldfield \& Reznick. Journal of Child Language, 22(2), 461-468.

Mervis, C.B., \& Bertrand, J. (1994). Acquisition of the Novel Name ${ }^{\wedge}$ Nameless Category (N3C) principle. Child Development, 65(6), 1646-1662.

Namy, L. L., \& Gentner, D. (2002). Making a silk purse out of a sow's ears: young children's use of comparison in category learning. Journal of Experimental Psychology. General, 131, 5-15. doi: 10.1037/0096-3445.131.1.5

Nelson, K. (1973). Structure and strategy in learning to talk. Monographs of the Society for Research in Child Development, 38(1-2). doi:10.2307/1165788

Quine, W. V. O. (1960). Word and Object. Cambridge, MA: MIT Press.

Rice, N. (1980). Cognition to language. Baltimore, MD: University Park Press.

Russell, E. E., \& Kyger, M. (under review). Animals, foods, and household items-oh my! Evidence of 24-30-month-old children's increasing flexibility in word learning from naturalistic data. Submitted to Psychology of Language and Communication.

Samuelson, L., \& Smith, L.B. (1999). Early noun vocabularies: Do ontology, category structure, and syntax correspond? Cognition, 71, 1-33.

Samuelson, L. K., \& Horst, J. S. (2007). Dynamic noun generalization: moment-tomoment interactions shape children's naming biases. Infancy, 11(1), 97-110. 
doi:10.1207/s15327078in1101_5

Samuelson, L. K., Horst, J. S., Schutte, A. R., \& Dobbertin, B. N. (2008). Rigid thinking about deformables: Do children sometimes overgeneralize the shape bias?. Journal of Child Language, 35(3), 559-589. doi:10.1017/ S0305000908008672

Sandhofer, C. M., Smith, L. B., \& Luo, J. (2000). Counting nouns and verbs in the input: Differential frequencies, different kinds of learning? Journal of Child Language, 27, 561-585.

Sandhofer, C., \& Smith, L. B. (2007). Learning adjectives in the real world: How learning nouns impedes learning adjectives. Language Learning and Development, 3(3), 233-267. doi:10.1080/15475440701360465

Santos, L. R., Hauser, M. D., \& Spelke, E. S. (2002). Domain-specific knowledge in human children and nonhuman primates: Artifacts and foods. In M. Bekoff, C. Allen, G. M. Burghardt, (Eds.), The cognitive animal: Empirical and theoretical perspectives on animal cognition (pp. 205-215). Cambridge, MA: MIT Press.

Shutts, K., Markson, L., \& Spelke, E. S. (2009). The developmental origins of animal and artifact concepts. In B. Hood \& L. Santos (Eds.), The origins of object knowledge (pp. 189-210). Oxford: Oxford University Press.

Smith, L. B. (2000). Learning how to learn words: An associative crane. In R. M. Golinkoff, K. Hirsh-Pasek, L. Bloom, L. Smith, A. Woodward, N. Akhtar, M. Tomasello, G. Hollich, Becoming a Word Learner: A Debate on Lexical Acquisition. New York, NY: Oxford University Press.

Smith, L. B. (2001) How domain-general processes may create domain-specific biases. In Bowerman, M. \& Levinson, S. (Eds.) Language Acquisition and Conceptual Development. Cambridge: Cambridge University Press.

Smith, L. B., \& Samuelson, L. (2006). An attentional learning account of the shape bias: Reply to Cimpian and Markman (2005) and Booth, Waxman, and Huang (2005). Developmental Psychology, 42(6), 1339-1343. doi:10.1037/0012-1649.42.6.1339

Smith, L.B., Jones, S.S., Landau, B, Gershkoff-Stowe, L., \& Samuelson, L. (2002). Object name learning provides on-the-job training for attention. Psychological Science. 13(1), 13-19.

Soja, N. (1992). Inferences about the meanings of nouns: The relationship between perceptual properties and syntax. Cognitive Development, 7, 29-46.

Soja, N. N., Carey, S., \& Spelke, E.S. (1991) Ontological categories guide young children's inductions of word meanings: Object terms and substance terms. Cognition, 38, 179-211.

Subrahmanyam, K., Landau, B., \& Gelman, R. (1999). Shape, material, and syntax: Interacting forces in children's learning of novel words for objects and substances. Language and Cognitive Processes, 14(3), 249-281. doi:10.1080/016909699386301

Tan, S. H., \& Schafer, G. (2005). Toddlers' novel word learning: Effects 
of phonological representation, vocabulary size and parents' ostensive behaviour. First Language, 25(2), 131-155. doi:10.1177/0142723705050338 Thom, E. E., \& Sandhofer, C. M. (2009). More is more: The relationship between vocabulary size and word extension. Journal of Experimental Child Psychology, 104(4), 466-473.

Waxman, S. R. (1994). The development of an appreciation of specific linkages between linguistic and conception organization. In L. R. Gleitman, \& B. L. Landau (Eds.). The acquisition of the lexicon (pp. 229-250). Cambridge, MA: MIT Press.

Woodward, A. L., Markman, E. M., \& Fitzsimmons, C. M. (1994). Rapid word learning in 13- and 18-month-olds. Developmental Psychology, 30, 553-556. 


\section{Appendix A}

Supplemental vocabulary checklist for Studies 1 and 2. Note that "foods" were eliminated for Study 2.

Dear parents,

Thank you for taking the time to participate in our study! This vocabulary questionnaire will help us better understand your child's language background.

Please mark the words your child comprehends and produces.

\begin{tabular}{l}
\hline Animals \\
Comprehends \\
\hline Alligator \\
Armadillo \\
Butterfly \\
Cougar (or mountain lion) \\
Dolphin \\
Giraffe \\
Gopher \\
Moth \\
Monkey \\
Octopus \\
Otter \\
Parrot \\
Panda \\
Prairie dog \\
Porpoise \\
Ram \\
Seahorse \\
Scorpion \\
Tortoise \\
Turtle
\end{tabular}


Wolf

Zebra

\section{Clothing items}

Bonnet

Beanie

Bootie

Barrette

Ear muffs

Goggles

Headband

Jumper

Mitten

Nose plug

Sunglasses

Sandals

Thongs (or flip flops)

Scarf

Foods

Apricots

Applesauce

Couscous

Gummies

Lentils

Noodles

Pasta

Pretzels

Peanut butter

Sunglasses

Rice

Tapioca

Wheat 